\title{
The Origin of Cooperativity in Double-Wheel Receptors. Freezing of Internal Rotation or Ligand Induced Torsional Strain?
}

\author{
Gianfranco Ercolani \\ Dipartimento di Scienze e Tecnologie Chimiche, Università di Roma Tor Vergata, Via della Ricerca \\ Scientifica,00133Roma, Italy.E-mail: ercolani@uniroma2.it \\ Supporting Information
}

Estimation of $\boldsymbol{V}_{\mathbf{0}}$ for $1 \mathrm{a}$. The value of $V_{0}$ for $\mathbf{1 a}$ has not been directly determined yet, but an estimate can be obtained by experiments carried out on the parent compound $\mathbf{1 b} .^{11}$ The steric crowding in $\mathbf{1 b}$ is basically the same as that in receptor 1a with the advantage that the internal rotation, interconverting two diastereomeric atropisomers, can be monitored by variabletemperature ${ }^{1} \mathrm{H}$ NMR spectroscopy and HPLC. The atropisomers have been separated by HPLC at $25^{\circ} \mathrm{C}$, and shown to undergo complete re-equilibration to the initial mixture upon standing at $20^{\circ} \mathrm{C}$ for $3 \mathrm{~h}^{11}$ Thus a conservative estimate for the half-life of the process is not less than $500 \mathrm{~s}$ (about one twentieth of the observed equilibration time), yielding $\mathrm{k} \approx 1.4 \times 10^{-3} \mathrm{~s}^{-1}$ and $\Delta G^{\$}{ }_{293} \approx 21 \mathrm{kcal}$ $\mathrm{mol}^{-1}$. This estimate is consistent with the non-coalescence of the $\beta$-pyrrole proton peaks up to 150 ${ }^{\circ} \mathrm{C}\left(400 \mathrm{MHz}{ }^{1} \mathrm{H} \mathrm{NMR}\right)$ indicating that the porphyrin rings rotate at rates much slower than ${ }^{1} \mathrm{H}$ NMR time-scale. ${ }^{11, \mathrm{~S} 1}$ Typical $\Delta S^{\ddagger}$ values for the internal rotation in cerium(IV) bis(mesotetraarylporphyrinate) complexes are in the range $-16,-23 \mathrm{cal} \mathrm{mol}^{-1} \mathrm{~K}^{-1}{ }^{3 b, \mathrm{~S} 1}$ Such a large negative activation entropy is probably due to the steric hindrance in the transition state between the eclipsed meso aryl groups which prevents their rotation. By assuming an average value of $\Delta S^{\dagger} \approx-20$ cal $\mathrm{mol}^{-1} \mathrm{~K}^{-1}$ for the internal rotation of $1 \mathrm{a}$, a value of $\Delta H^{\ddagger} \approx 15 \mathrm{kcal} \mathrm{mol}^{-1}$ can be calculated. The latter represents to a good approximation the height of the potential energy barrier, $V_{0}$.

Calculation of the Reduced Moment of Inertia of 1a. Optimization of the geometry of the staggered conformation of 1 a $\left(D_{4 d}\right.$ symmetry) at the B3LYP/6-31G(d) (C, H, N), SDD (Ce) level of theory (vide infra) gave the following rotational constants:

$\begin{array}{llll}\text { Rotational constants (GHZ) : } & 0.0217101 & 0.0217101 & 0.0154138\end{array}$

The principal moments of inertia $\left(I_{x}, I_{y}, I_{z}\right)$ of the molecule depend on the rotational constants $\left(R_{x}\right.$, $R_{y}, R_{z}$ ) according to eq $\mathrm{S} 1$ 


$$
I_{i}=\frac{h}{8 \pi^{2} R_{i}}
$$

where $h$, the Planck constant, is measured in $\mathrm{J} \mathrm{s}, R_{i}$ in $\mathrm{Hz}$, and $I_{i}$ in $\mathrm{kg} \mathrm{\textrm {m } ^ { 2 }}$. By symmetry considerations, since $I_{x}=I_{y}$, it is evident the the axis about which $I_{z}$ is measured, coincides with the axis of internal rotation. Receptor 1a consists of two coaxial symmetric tops of $C_{4}$ symmetry, thus the moment of inertia of each top about the rotational axis is $I_{z} / 2$. In the case of two coaxial symmetric tops the reduced moment of inertia is

$$
I_{r}=\frac{I_{1} I_{2}}{I_{1}+I_{2}}
$$

where $I_{1}$ and $I_{2}$ are the moments of inertia of the two groups about the axis of internal rotation. ${ }^{10}$ Since in the present case $I_{1}=I_{2}=I_{z} / 2$, the reduced moment of inertia is $I_{r}=I_{z} / 4$.

Derivation of Eq 3. As illustrated in Scheme 1, the stepwise association constants for the binding of the substrate $\mathrm{S}$ to the receptor $1 \mathrm{a}$ are the following: $K_{1}=4 K / L ; K_{2}=3 K / 2 ; K_{3}=2 K / 3 ; K_{4}=K / 4$, where $K / L$ and $K$ are the microscopic association constants for the binding of the first ligand and the successive ligands, respectively, and where the factors multiplying them are statistical factors given by $(t-n+1) / n$ where $t$ is the total number of sites per wheel and $n$ is the subscript of the corresponding stepwise association constant. ${ }^{7 \mathrm{a}}$ The occupancy, $r$, defined as the average number of ligands bound to the receptor, is given, in the case $t=4$, by eq S3

$$
r=\frac{K_{1}[\mathrm{~S}]+2 K_{1} K_{2}[\mathrm{~S}]^{2}+3 K_{1} K_{2} K_{3}[\mathrm{~S}]^{3}+4 K_{1} K_{2} K_{3} K_{4}[\mathrm{~S}]^{4}}{1+K_{1}[\mathrm{~S}]+K_{1} K_{2}[\mathrm{~S}]^{2}+K_{1} K_{2} K_{3}[\mathrm{~S}]^{3}+K_{1} K_{2} K_{3} K_{4}[\mathrm{~S}]^{4}}
$$

where $[\mathrm{S}]$ is the free substrate concentration. ${ }^{7 \mathrm{a}}$ Substitution of the stepwise association constants, as defined above, into eq S3, affords eq S4

$$
\begin{aligned}
r & =\frac{4 K[\mathrm{~S}]+12 K^{2}[\mathrm{~S}]^{2}+12 K^{3}[\mathrm{~S}]^{3}+4 K^{4}[\mathrm{~S}]^{4}}{L+4 K[\mathrm{~S}]+6 K^{2}[\mathrm{~S}]^{2}+4 K^{3}[\mathrm{~S}]^{3}+K^{4}[\mathrm{~S}]^{4}} \\
& =\frac{4 K[S](1+K[S])^{3}}{L-1+(1+K[S])^{4}}
\end{aligned}
$$


Considering that the degree of saturation, $Y$, is defined as $r / t$, eq 3 is immediately obtained.

Derivation of the Plot in Figure 1. A standard measure of cooperativity is the Hill coefficient at half-saturation, defined by eq 4 . Strictly speaking, the Hill coefficient does not always have its maximum exactly at half-saturation, however, as pointed out by Perlmutter-Hayman, ${ }^{7 \mathrm{a}}$ since the Hill plot is very nearly straight over a wide range of $r$, the value of $n_{\mathrm{H}}$ estimated at half-saturation coincides with $\left(n_{\mathrm{H}}\right)_{\max }$ for all practical purposes.

Defining $\alpha=K[\mathrm{~S}]$, and considering that decimal logarithms are proportional to natural logarithms and that $d \ln x=d x / x$, eq 4 is easily transformed into eq S5

$$
n_{\mathrm{H}}=\left(\alpha \frac{d[Y /(1-Y)]}{d \alpha}\right)_{Y=1 / 2}
$$

From eq 3, eq S6 is easily obtained

$$
\frac{Y}{1-Y}=\frac{\alpha(1+\alpha)^{3}}{L-1+(1+\alpha)^{3}}
$$

The derivative of eq S6 with respect to $\alpha$ yields eq S7.

$$
\frac{d[Y /(1-Y)]}{d \alpha}=\frac{(1+\alpha)^{2}\left(L+4 L \alpha+6 \alpha^{2}+4 \alpha^{3}+\alpha^{4}\right)}{\left(L+3 \alpha+3 \alpha^{2}+\alpha^{3}\right)^{2}}
$$

Substitution of eq S7 into eq S5 yields eq S8

$$
n_{\mathrm{H}}=\frac{\alpha_{1 / 2}\left(1+\alpha_{1 / 2}\right)^{2}\left(L+4 L \alpha_{1 / 2}+6 \alpha_{1 / 2}^{2}+4 \alpha_{1 / 2}^{3}+\alpha_{1 / 2}^{4}\right)}{\left(L+3 \alpha_{1 / 2}+3 \alpha_{1 / 2}^{2}+\alpha_{1 / 2}^{3}\right)^{2}}
$$

where $\alpha_{1 / 2}$ is the value of $\alpha$ at half-saturation.

Solution of eq S6 with $Y=1 / 2$ has been carried out with the help of a software for symbolic computation yielding eq S9 


$$
\alpha_{1 / 2}=\frac{R-3}{6}+\sqrt{\frac{1}{2}-\frac{Q}{12}+\frac{L-1}{Q}+\frac{3}{2 R}}
$$

where

$$
Q=\left[-54(L-1)+6 \sqrt{81(L-1)^{2}+48(L-1)^{3}}\right]^{1 / 3}
$$

and

$$
R=\sqrt{9+3 Q-\frac{36(L-1)}{Q}}
$$

It should be noted that $\alpha_{1 / 2}$ only depends on the allosteric constant $L$.

Substitution of eq S9 into eq S8 allows the calculation of $n_{\mathrm{H}}$ as a function of $L$. The result is plotted in Figure 1.

\section{DFT calculations.}

1. Optimized geometry of the staggered conformation of $1 \mathbf{a}\left(D_{4 \mathrm{~d}}\right.$ symmetry) at the B3LYP/6-31G(d) (C, H, N), SDD (Ce) level of theory by the Gaussian 03 program.

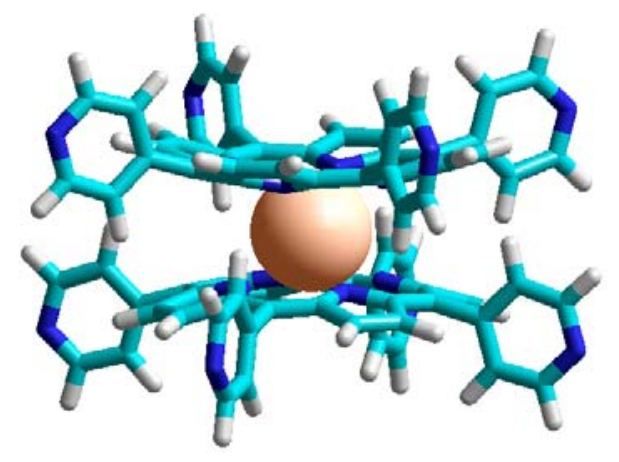


$\begin{array}{llll}6 & -2.488641 & -3.450758 & 2.087095\end{array}$

$\begin{array}{lllll}1 & -2.593858 & -4.491740 & 2.356591\end{array}$

$\begin{array}{llll}6 & -3.450758 & -2.488641 & 2.087095\end{array}$

$\begin{array}{lllll}1 & -4.491740 & -2.593858 & 2.356591\end{array}$

$6-2.804156-1.248311 \quad 1.722536$

$\begin{array}{lllll}6 & 0.000000 & -3.444130 & 1.799759\end{array}$

$\begin{array}{lllll}6 & 0.000000 & -4.896606 & 2.189979\end{array}$

$\begin{array}{llll}6 & 0.000000 & -5.276283 & 3.539500\end{array}$

$\begin{array}{lllll}1 & 0.000000 & -4.524211 & 4.323114\end{array}$

$\begin{array}{llll}6 & 0.000000 & -6.633275 & 3.864097\end{array}$

$\begin{array}{lllll}1 & 0.000000 & -6.944903 & 4.907592\end{array}$

$\begin{array}{lllll}7 & 0.000000 & -7.617544 & 2.955852\end{array}$

$\begin{array}{lllll}6 & 0.000000 & -7.249039 & 1.669346\end{array}$

$\begin{array}{lllll}1 & 0.000000 & -8.058453 & 0.940694\end{array}$

$\begin{array}{lllll}6 & 0.000000 & -5.920802 & 1.236701\end{array}$

$\begin{array}{lllll}1 & 0.000000 & -5.685703 & 0.177861\end{array}$

$\begin{array}{lllll}7 & 1.469214 & -1.469214 & 1.452510\end{array}$

$\begin{array}{lllll}6 & 2.804156 & -1.248311 & 1.722536\end{array}$

$\begin{array}{lllll}6 & 3.450758 & -2.488641 & 2.087095\end{array}$

$\begin{array}{lllll}1 & 4.491740 & -2.593858 & 2.356591\end{array}$

$\begin{array}{lllll}6 & 2.488641 & -3.450758 & 2.087095\end{array}$

$\begin{array}{lllll}1 & 2.593858 & -4.491740 & 2.356591\end{array}$

$\begin{array}{lllll}6 & 1.248311 & -2.804156 & 1.722536\end{array}$

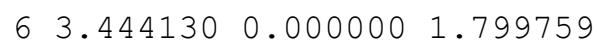

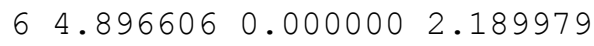

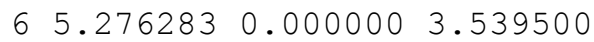

$\begin{array}{lllll}1 & 4.524211 & 0.000000 & 4.323114\end{array}$

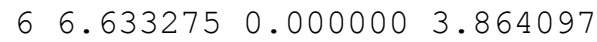

$16.944903 \quad 0.000000 \quad 4.907592$

$\begin{array}{lllll}7 & 7.617544 & 0.000000 & 2.955852\end{array}$

$\begin{array}{llll}67.249039 & 0.000000 & 1.669346\end{array}$

$18.058453 \quad 0.000000 \quad 0.940694$

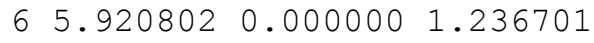

$\begin{array}{lllll}1 & 5.685703 & 0.000000 & 0.177861\end{array}$

$\begin{array}{lllll}6 & -3.444130 & 0.000000 & 1.799759\end{array}$

$\begin{array}{lllll}6 & -4.896606 & 0.000000 & 2.189979\end{array}$

$\begin{array}{llll}6 & -5.276283 & 0.000000 & 3.539500\end{array}$

$\begin{array}{lllll}1 & -4.524211 & 0.000000 & 4.323114\end{array}$

$\begin{array}{llll}6 & -6.633275 & 0.000000 & 3.864097\end{array}$

$\begin{array}{lllll}1 & -6.944903 & 0.000000 & 4.907592\end{array}$

$\begin{array}{lllll}7 & -7.617544 & 0.000000 & 2.955852\end{array}$

$\begin{array}{lllll}6 & -7.249039 & 0.000000 & 1.669346\end{array}$

$1-8.058453 \quad 0.000000 \quad 0.940694$

$\begin{array}{lllll}6 & -5.920802 & 0.000000 & 1.236701\end{array}$

$\begin{array}{llllll}1 & -5.685703 & 0.000000 & 0.177861\end{array}$

$\begin{array}{lllll}6 & 2.435368 & 2.435368 & -1.799759\end{array}$

$\begin{array}{lllll}6 & 3.462424 & 3.462424 & -2.189979\end{array}$

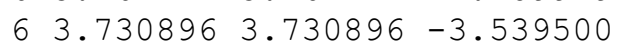

$\begin{array}{lllll}1 & 3.199100 & 3.199100 & -4.323114\end{array}$

$\begin{array}{lllll}6 & 4.690434 & 4.690434 & -3.864097\end{array}$

$\begin{array}{lllll}1 & 4.910788 & 4.910788 & -4.907592\end{array}$

$\begin{array}{lllll}7 & 5.386417 & 5.386417 & -2.955852\end{array}$

$\begin{array}{lllll}6 & 5.125844 & 5.125844 & -1.669346\end{array}$

$15.698187 \quad 5.698187-0.940694$

$\begin{array}{lllll}6 & 4.186640 & 4.186640 & -1.236701\end{array}$

$14.0203994 .020399-0.177861$

$\begin{array}{lllll}7 & 0.000000 & 2.077782 & -1.452510\end{array}$

$\begin{array}{llll}6 & -1.100149 & 2.865527 & -1.722536\end{array}$

$\begin{array}{lllll}6 & -0.680319 & 4.199790 & -2.087095\end{array}$
$1-1.342005 \quad 5.010275-2.356591$

$\begin{array}{lllll}6 & 0.680319 & 4.199790 & -2.087095\end{array}$

$11.342005 \quad 5.010275-2.356591$

$\begin{array}{lllll}6 & 1.100149 & 2.865527 & -1.722536\end{array}$

$\begin{array}{lllll}6 & -2.435368 & 2.435368 & -1.799759\end{array}$

$\begin{array}{lllll}6 & -3.462424 & 3.462424 & -2.189979\end{array}$

$6-3.730896 \quad 3.730896-3.539500$

$1-3.199100 \quad 3.199100-4.323114$

$\begin{array}{lllll}6 & -4.690434 & 4.690434 & -3.864097\end{array}$

$\begin{array}{lllll}1 & -4.910788 & 4.910788 & -4.907592\end{array}$

$\begin{array}{lllll}7 & -5.386417 & 5.386417 & -2.955852\end{array}$

$\begin{array}{lllll}6 & -5.125844 & 5.125844 & -1.669346\end{array}$

$1-5.698187 \quad 5.698187-0.940694$

$6-4.186640 \quad 4.186640-1.236701$

$1-4.0203994 .020399-0.177861$

$6-2.435368-2.435368-1.799759$

$6-3.462424-3.462424-2.189979$

$6-3.730896-3.730896-3.539500$

$1-3.199100-3.199100-4.323114$

$\begin{array}{lllll}6 & -4.690434 & -4.690434 & -3.864097\end{array}$

$1-4.910788-4.910788-4.907592$

$\begin{array}{lllll}7 & -5.386417 & -5.386417 & -2.955852\end{array}$

$6-5.125844-5.125844-1.669346$

$1-5.698187-5.698187-0.940694$

$6-4.186640-4.186640-1.236701$

$1-4.020399-4.020399-0.177861$

$70.000000-2.077782-1.452510$

$\begin{array}{lllll}6 & 1.100149 & -2.865527 & -1.722536\end{array}$

$60.680319-4.199790-2.087095$

$11.342005-5.010275-2.356591$

$6-0.680319-4.199790-2.087095$

$1-1.342005-5.010275-2.356591$

$6-1.100149-2.865527-1.722536$

$\begin{array}{lllll}6 & 2.435368 & -2.435368 & -1.799759\end{array}$

$\begin{array}{lllll}6 & 3.462424 & -3.462424 & -2.189979\end{array}$

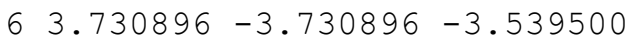

$13.199100-3.199100-4.323114$

$\begin{array}{lllll}6 & 4.690434 & -4.690434 & -3.864097\end{array}$

$14.910788-4.910788-4.907592$

$\begin{array}{lllll}7 & 5.386417 & -5.386417 & -2.955852\end{array}$

$\begin{array}{lllll}6 & 5.125844 & -5.125844 & -1.669346\end{array}$

$15.698187-5.698187-0.940694$

$\begin{array}{lllll}6 & 4.186640 & -4.186640 & -1.236701\end{array}$

$14.020399-4.020399-0.177861$

$\begin{array}{llll}7 & 2.077782 & 0.000000 & -1.452510\end{array}$

$\begin{array}{llll}6 & 2.865527 & 1.100149 & -1.722536\end{array}$

$\begin{array}{lllll}6 & 4.199790 & 0.680319 & -2.087095\end{array}$

$15.0102751 .342005-2.356591$

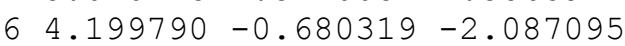

$15.010275-1.342005-2.356591$

$62.865527-1.100149-1.722536$

$7 \quad-2.077782 \quad 0.000000-1.452510$

$6-2.865527-1.100149-1.722536$

$6-4.199790-0.680319-2.087095$

$1-5.010275-1.342005-2.356591$

$6-4.199790 \quad 0.680319-2.087095$

$1-5.010275 \quad 1.342005-2.356591$

$\begin{array}{lllll}6 & -2.865527 & 1.100149 & -1.722536\end{array}$

$E(R B+H F-L Y P)=-4428.62950796 \quad$ A.U. 
2. Optimized geometry of the eclipsed conformation of 1a (torsional angle $\varphi$ fixed at $45^{\circ}$, $D_{4}$ symmetry) at the B3LYP/6-31G(d) (C, H, N), SDD (Ce) level of theory by the Gaussian 03 program.

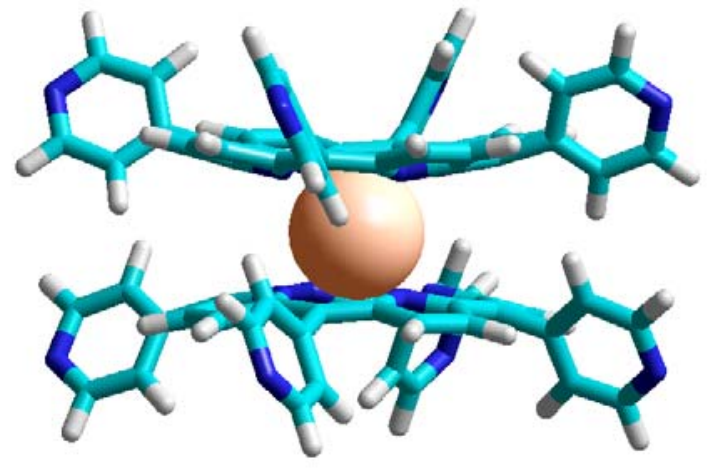

$580.000000 \quad 0.000000 \quad 0.000000$

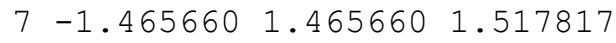

$\begin{array}{lllll}6 & -2.808124 & 1.236213 & 1.763104\end{array}$

$6-3.4771292 .4839852 .055854$

$\begin{array}{lll}1-4.530057 & 2.601936 & 2.262060\end{array}$

$\begin{array}{llll}6 & -2.523170 & 3.448978 & 2.077809\end{array}$

$\begin{array}{lllll}1 & -2.655727 & 4.491390 & 2.322117\end{array}$

$\begin{array}{lllll}6 & -1.266831 & 2.805053 & 1.762877\end{array}$

$\begin{array}{lllll}6 & -0.016764 & 3.445957 & 1.863817\end{array}$

$\begin{array}{llllll}6 & -0.044531 & 4.876606 & 2.315588\end{array}$

$\begin{array}{lllll}6 & 0.472831 & 5.252286 & 3.565465\end{array}$

$\begin{array}{lll}1 & 0.920765 \quad 4.511606 & 4.220209\end{array}$

$\begin{array}{lllll}6 & 0.392797 & 6.585189 & 3.968681\end{array}$

$\begin{array}{lllll}1 & 0.790017 & 6.884588 & 4.937373\end{array}$

$\begin{array}{llllll}7 & -0.160584 & 7.557870 & 3.233186\end{array}$

$\begin{array}{lllll}6 & -0.667582 & 7.196365 & 2.048547\end{array}$

$\begin{array}{lllll}1 & -1.120994 & 7.991216 & 1.458187\end{array}$

$\begin{array}{lllll}6 & -0.640624 & 5.891364 & 1.553587\end{array}$

$\begin{array}{lllll}1 & -1.079072 & 5.669055 & 0.587273\end{array}$

$\begin{array}{llll}7 & -1.465660 & -1.465660 & 1.517817\end{array}$

$6-1.236213-2.808124 \quad 1.763104$

$6-2.483985-3.4771292 .055854$

$1-2.601936-4.5300572 .262060$

$6-3.448978-2.523170 \quad 2.077809$

$\begin{array}{lllll}1 & -4.491390 & -2.655727 & 2.322117\end{array}$

$\begin{array}{llll}6 & -2.805053 & -1.266831 & 1.762877\end{array}$

$\begin{array}{lllll}6 & -3.445957 & -0.016764 & 1.863817\end{array}$

$\begin{array}{llll}6 & -4.876606 & -0.044531 & 2.315588\end{array}$

$\begin{array}{lllll}6 & -5.252286 & 0.472831 & 3.565465\end{array}$

$\begin{array}{lllll}1 & -4.511606 & 0.920765 & 4.220209\end{array}$

$\begin{array}{lllll}6 & -6.585189 & 0.392797 & 3.968681\end{array}$

$\begin{array}{lllll}1 & -6.884588 & 0.790017 & 4.937373\end{array}$

$\begin{array}{llll}7 & -7.557870 & -0.160584 & 3.233186\end{array}$

$6-7.196365-0.667582 \quad 2.048547$

$1-7.991216-1.120994 \quad 1.458187$

$\begin{array}{llll}6 & -5.891364 & -0.640624 & 1.553587\end{array}$

$1-5.669055-1.0790720 .587273$

$\begin{array}{llll}7 & 1.465660 & -1.465660 & 1.517817\end{array}$ $\begin{array}{lllll}6 & 2.808124 & -1.236213 & 1.763104\end{array}$

$\begin{array}{llll}6 & 3.477129 & -2.483985 & 2.055854\end{array}$

$14.530057-2.6019362 .262060$

$\begin{array}{llll}6 & 2.523170 & -3.448978 & 2.077809\end{array}$

$\begin{array}{lllll}1 & 2.655727 & -4.491390 & 2.322117\end{array}$

$\begin{array}{lllll}6 & 1.266831 & -2.805053 & 1.762877\end{array}$

$\begin{array}{lllll}6 & 0.016764 & -3.445957 & 1.863817\end{array}$

$\begin{array}{llllll}6 & 0.044531 & -4.876606 & 2.315588\end{array}$

$6-0.472831-5.252286 \quad 3.565465$

$1-0.920765-4.511606 \quad 4.220209$

$\begin{array}{lllll}6 & -0.392797 & -6.585189 & 3.968681\end{array}$

$\begin{array}{lllll}1 & -0.790017 & -6.884588 & 4.937373\end{array}$

$\begin{array}{lllll}7 & 0.160584 & -7.557870 & 3.233186\end{array}$

$\begin{array}{lllll}6 & 0.667582 & -7.196365 & 2.048547\end{array}$

$\begin{array}{lllll}1 & 1.120994 & -7.991216 & 1.458187\end{array}$

$\begin{array}{lllll}6 & 0.640624 & -5.891364 & 1.553587\end{array}$

$\begin{array}{llll}1 & 1.079072 & -5.669055 & 0.587273\end{array}$

$\begin{array}{lllll}7 & 1.465660 & 1.465660 & 1.517817\end{array}$

$\begin{array}{lllll}6 & 1.236213 & 2.808124 & 1.763104\end{array}$

$\begin{array}{lllll}6 & 2.483985 & 3.477129 & 2.055854\end{array}$

$12.601936 \quad 4.530057 \quad 2.262060$

$\begin{array}{llll}6 & 3.448978 & 2.523170 & 2.077809\end{array}$

$\begin{array}{lllll}1 & 4.491390 & 2.655727 & 2.322117\end{array}$

$\begin{array}{llll}6 & 2.805053 & 1.266831 & 1.762877\end{array}$

$\begin{array}{lllll}6 & 3.445957 & 0.016764 & 1.863817\end{array}$

$\begin{array}{lllll}6 & 4.876606 & 0.044531 & 2.315588\end{array}$

$\begin{array}{lllll}6 & 5.252286 & -0.472831 & 3.565465\end{array}$

$14.511606-0.9207654 .220209$

$6 \quad 6.585189-0.3927973 .968681$

$16.884588-0.790017 \quad 4.937373$

$\begin{array}{lllll}7 & 7.557870 & 0.160584 & 3.233186\end{array}$

$\begin{array}{lllll}6 & 7.196365 & 0.667582 & 2.048547\end{array}$

$\begin{array}{llll}1 & 7.991216 & 1.120994 & 1.458187\end{array}$

$\begin{array}{lllll}6 & 5.891364 & 0.640624 & 1.553587\end{array}$

15.6690551 .0790720 .587273

$7-1.465660 \quad 1.465660-1.517817$

$\begin{array}{lllll}6 & -1.236213 & 2.808124 & -1.763104\end{array}$

$\begin{array}{lllll}6 & -2.483985 & 3.477129 & -2.055854\end{array}$ 
$\begin{array}{lllll}1 & -2.601936 & 4.530057 & -2.262060\end{array}$

$\begin{array}{llll}6 & -3.448978 & 2.523170 & -2.077809\end{array}$

$\begin{array}{lllll}1 & -4.491390 & 2.655727 & -2.322117\end{array}$

$\begin{array}{llll}6 & -2.805053 & 1.266831 & -1.762877\end{array}$

$\begin{array}{lllll}6 & -3.445957 & 0.016764 & -1.863817\end{array}$

$\begin{array}{lllll}6 & -4.876606 & 0.044531 & -2.315588\end{array}$

$6-5.252286-0.472831-3.565465$

$1-4.511606-0.920765-4.220209$

$6-6.585189-0.392797-3.968681$

$1-6.884588-0.790017-4.937373$

$\begin{array}{lllll}7 & -7.557870 & 0.160584 & -3.233186\end{array}$

$\begin{array}{llll}6 & -7.196365 & 0.667582 & -2.048547\end{array}$

$\begin{array}{lllll}1 & -7.991216 & 1.120994 & -1.458187\end{array}$

$\begin{array}{lllll}6 & -5.891364 & 0.640624 & -1.553587\end{array}$

$\begin{array}{lllll}1 & -5.669055 & 1.079072 & -0.587273\end{array}$

$\begin{array}{llll}7 & 1.465660 & 1.465660 & -1.517817\end{array}$

$\begin{array}{llll}6 & 2.808124 & 1.236213 & -1.763104\end{array}$

$\begin{array}{llll}6 & 3.477129 & 2.483985 & -2.055854\end{array}$

$\begin{array}{lllll}1 & 4.530057 & 2.601936 & -2.262060\end{array}$

$\begin{array}{lllll}6 & 2.523170 & 3.448978 & -2.077809\end{array}$

$\begin{array}{lllll}1 & 2.655727 & 4.491390 & -2.322117\end{array}$

$\begin{array}{lllll}6 & 1.266831 & 2.805053 & -1.762877\end{array}$

$\begin{array}{llllll}6 & 0.016764 & 3.445957 & -1.863817\end{array}$

$\begin{array}{llllll}6 & 0.044531 & 4.876606 & -2.315588\end{array}$

$6-0.4728315 .252286-3.565465$

$1-0.920765 \quad 4.511606-4.220209$

$\begin{array}{llll}6 & -0.392797 & 6.585189 & -3.968681\end{array}$

$\begin{array}{lllll}1 & -0.790017 & 6.884588 & -4.937373\end{array}$

$\begin{array}{lllll}7 & 0.160584 & 7.557870 & -3.233186\end{array}$

$\begin{array}{lllll}6 & 0.667582 & 7.196365 & -2.048547\end{array}$

$11.120994 \quad 7.991216-1.458187$

$\begin{array}{lllll}6 & 0.640624 & 5.891364 & -1.553587\end{array}$

$11.0790725 .669055-0.587273$

$\begin{array}{lllll}7 & 1.465660 & -1.465660 & -1.517817\end{array}$

$61.236213-2.808124-1.763104$
$62.483985-3.477129-2.055854$

$12.601936-4.530057-2.262060$

$63.448978-2.523170-2.077809$

$\begin{array}{lllll}1 & 4.491390 & -2.655727 & -2.322117\end{array}$

$\begin{array}{lllll}6 & 2.805053 & -1.266831 & -1.762877\end{array}$

$\begin{array}{lllll}6 & 3.445957 & -0.016764 & -1.863817\end{array}$

$\begin{array}{lllll}6 & 4.876606 & -0.044531 & -2.315588\end{array}$

$\begin{array}{lllll}6 & 5.252286 & 0.472831 & -3.565465\end{array}$

$14.511606 \quad 0.920765-4.220209$

$\begin{array}{llllll}6 & 6.585189 & 0.392797 & -3.968681\end{array}$

$16.884588 \quad 0.790017-4.937373$

$77.557870-0.160584-3.233186$

$67.196365-0.667582-2.048547$

$17.991216-1.120994-1.458187$

$\begin{array}{lllll}6 & 5.891364 & -0.640624 & -1.553587\end{array}$

$15.669055-1.079072-0.587273$

$7-1.465660-1.465660-1.517817$

$6-2.808124-1.236213-1.763104$

$6-3.477129-2.483985-2.055854$

$1-4.530057-2.601936-2.262060$

$6-2.523170-3.448978-2.077809$

$\begin{array}{lllll}1 & -2.655727 & -4.491390 & -2.322117\end{array}$

$6-1.266831-2.805053-1.762877$

$6-0.016764-3.445957-1.863817$

$6-0.044531-4.876606-2.315588$

$\begin{array}{lllll}6 & 0.472831 & -5.252286 & -3.565465\end{array}$

$10.920765-4.511606-4.220209$

$\begin{array}{lllll}6 & 0.392797 & -6.585189 & -3.968681\end{array}$

$\begin{array}{lllll}1 & 0.790017 & -6.884588 & -4.937373\end{array}$

$7-0.160584-7.557870-3.233186$

$6-0.667582-7.196365-2.048547$

$1-1.120994-7.991216-1.458187$

$6-0.640624-5.891364-1.553587$

$1-1.079072-5.669055-0.587273$

$E(R B+H F-L Y P)=-4428.59989175 \quad$ A.U.

3. Optimized geometry of the the complex 1a・(2) $)_{4}\left(D_{4}\right.$ symmetry) at the B3LYP/6-31G(d) (C, H, N, O), SDD (Ce) level of theory by the Gaussian 03 program.

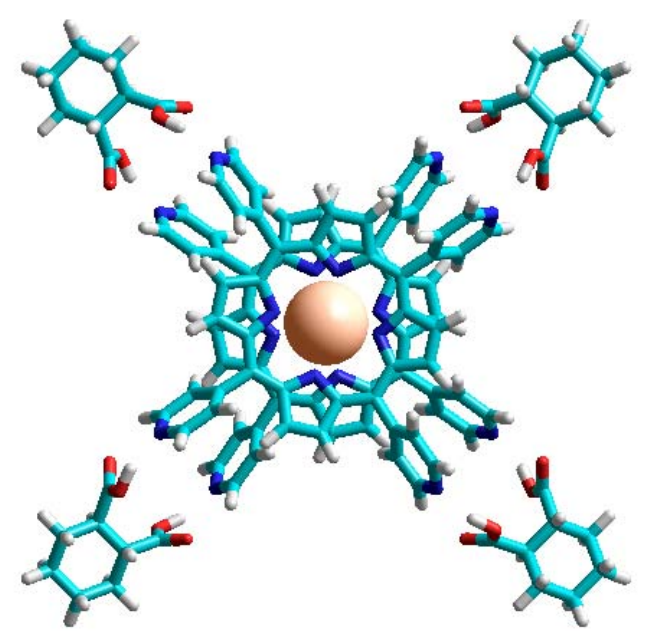


$\begin{array}{lllll}1 & 1.354317 & 9.389939 & 2.121451\end{array}$

$\begin{array}{llll}1 & -1.354317 & 9.389939 & -2.121451\end{array}$

$\begin{array}{lllll}6 & 0.775738 & 11.749100 & -0.047452\end{array}$

$\begin{array}{lllll}6 & 1.347723 & 13.020400 & 0.618478\end{array}$

$\begin{array}{llllll}6 & 0.767618 & 14.291390 & -0.019510\end{array}$

$\begin{array}{lllll}6 & 1.383987 & 10.487010 & 0.547350\end{array}$

$\begin{array}{lllll}8 & 2.112699 & 9.736051 & -0.079622\end{array}$

$\begin{array}{llll}8 & 1.030077 & 10.288850 & 1.823800\end{array}$

$11.05968911 .738300-1.104470$

$12.441462 \quad 13.017140 \quad 0.530782$

$11.113883 \quad 12.998210 \quad 1.690695$

$11.10426914 .357730 \quad-1.064258$

$\begin{array}{lllll}1 & 1.161172 & 15.178090 & 0.493291\end{array}$

$\begin{array}{lllll}6 & -0.775738 & 11.749100 & 0.047452\end{array}$

$\begin{array}{lllll}6 & -1.347723 & 13.020400 & -0.618478\end{array}$

$\begin{array}{lllll}6 & -0.767618 & 14.291390 & 0.019510\end{array}$

$\begin{array}{lllll}6 & -1.383987 & 10.487010 & -0.547350\end{array}$

$\begin{array}{lllll}8 & -2.112699 & 9.736051 & 0.079622\end{array}$

$8-1.030077 \quad 10.288850-1.823800$

$\begin{array}{llll}1 & -1.059689 & 11.738300 & 1.104470\end{array}$

$1-2.441462 \quad 13.017140-0.530782$

$\begin{array}{lllll}1 & -1.113883 & 12.998210 & -1.690695\end{array}$

$1-1.10426914 .3577301 .064258$

$\begin{array}{lllll}1 & -1.161172 & 15.178090 & -0.493291\end{array}$

$1-9.389939-1.354317-2.121451$

$6-11.749100 \quad 0.775738-0.047452$

$\begin{array}{llll}6 & -13.020400 & 1.347723 & 0.618478\end{array}$

$\begin{array}{lllll}6 & -14.291390 & 0.767618 & -0.019510\end{array}$

$\begin{array}{lllll}6 & -10.487010 & 1.383987 & 0.547350\end{array}$

$\begin{array}{lllll}8 & -9.736051 & 2.112699 & -0.079622\end{array}$

$8-10.288850 \quad 1.030077 \quad 1.823800$

$1-11.738300 \quad 1.059689-1.104470$

$\begin{array}{lllll}1 & -13.017140 & 2.441462 & 0.530782\end{array}$

$1-12.998210 \quad 1.1138831 .690695$

$1-14.357730 \quad 1.104269-1.064258$

$\begin{array}{lllll}1 & -15.178090 & 1.161172 & 0.493291\end{array}$

$\begin{array}{lllll}6 & -11.749100 & -0.775738 & 0.047452\end{array}$

$6-13.020400-1.347723-0.618478$

$\begin{array}{lllll}6 & -14.291390 & -0.767618 & 0.019510\end{array}$

$6-10.487010-1.383987-0.547350$

$8-9.736051-2.1126990 .079622$

$8-10.288850-1.030077-1.823800$

$1-11.738300-1.0596891 .104470$

$1-13.017140-2.441462-0.530782$

$1-12.998210-1.113883-1.690695$

$1-14.357730-1.1042691 .064258$

$1-15.178090-1.161172-0.493291$

$\begin{array}{llll}1 & -9.389939 & 1.354317 & 2.121451\end{array}$

$\begin{array}{llll}6 & -1.809621 & -2.480989 & 1.730578\end{array}$

$\begin{array}{lllll}6 & -3.161113 & -2.861150 & 2.069628\end{array}$

$\begin{array}{lllll}1 & -3.489626 & -3.860221 & 2.315075\end{array}$

$\begin{array}{llll}7 & 1.121267 & -1.741398 & 1.499371\end{array}$

$\begin{array}{lllll}6 & 1.721715 & -3.901948 & 2.079044\end{array}$

$\begin{array}{lllll}1 & 1.626222 & -4.948322 & 2.325966\end{array}$

$\begin{array}{lllll}6 & 0.633254 & -3.004957 & 1.754796\end{array}$

$\begin{array}{lllll}6 & -0.726054 & -3.374223 & 1.802984\end{array}$

$\begin{array}{lllll}6 & -1.043100 & -4.818195 & 2.056403\end{array}$

$6-0.696230-5.468453 \quad 3.251149$

$1-0.222486-4.923160 \quad 4.061255$

$6-0.948411-6.832948 \quad 3.383291$

$\begin{array}{lllll}1 & -0.666505 & -7.358997 & 4.292671\end{array}$ $\begin{array}{lllll}7 & -1.523327 & -7.572701 & 2.424179\end{array}$

$\begin{array}{lllll}6 & -1.903321 & -6.945793 & 1.300022\end{array}$

$\begin{array}{lllll}1 & -2.354493 & -7.582548 & 0.544102\end{array}$

$\begin{array}{lllll}6 & -1.691076 & -5.586194 & 1.080744\end{array}$

$1-2.002208-5.132362 \quad 0.146956$

$\begin{array}{llll}6 & 2.480989 & -1.809621 & 1.730578\end{array}$

$\begin{array}{llll}6 & 2.861150 & -3.161113 & 2.069628\end{array}$

$\begin{array}{llll}1 & 3.860221 & -3.489626 & 2.315075\end{array}$

$\begin{array}{lllll}7 & 1.741398 & 1.121267 & 1.499371\end{array}$

$\begin{array}{llll}6 & 3.901948 & 1.721715 & 2.079044\end{array}$

$\begin{array}{llll}1 & 4.948322 & 1.626222 & 2.325966\end{array}$

$\begin{array}{llll}6 & 3.004957 & 0.633254 & 1.754796\end{array}$

$\begin{array}{lllll}6 & 3.374223 & -0.726054 & 1.802984\end{array}$

$\begin{array}{lll}6 & 4.818195-1.043100 & 2.056403\end{array}$

$\begin{array}{llll}6 & 5.468453-0.696230 & 3.251149\end{array}$

$14.923160-0.222486 \quad 4.061255$

$\begin{array}{lllll}6 & 6.832948 & -0.948411 & 3.383291\end{array}$

$17.358997-0.666505 \quad 4.292671$

$\begin{array}{lllll}7 & 7.572701 & -1.523327 & 2.424179\end{array}$

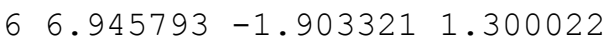

$17.582548-2.354493 \quad 0.544102$

$\begin{array}{lllll}6 & 5.586194 & -1.691076 & 1.080744\end{array}$

$15.132362-2.002208 \quad 0.146956$

$\begin{array}{llll}6 & 1.809621 & 2.480989 & 1.730578\end{array}$

$\begin{array}{llllll}6 & 3.161113 & 2.861150 & 2.069628\end{array}$

$\begin{array}{lllll}1 & 3.489626 & 3.860221 & 2.315075\end{array}$

$\begin{array}{llll}7 & -1.121267 & 1.741398 & 1.499371\end{array}$

$\begin{array}{lllll}6 & -1.721715 & 3.901948 & 2.079044\end{array}$

$\begin{array}{llll}1 & -1.626222 & 4.948322 & 2.325966\end{array}$

$\begin{array}{lllll}6 & -0.633254 & 3.004957 & 1.754796\end{array}$

$\begin{array}{lllll}6 & 0.726054 & 3.374223 & 1.802984\end{array}$

$61.043100 \quad 4.8181952 .056403$

$\begin{array}{llll}6 & 0.696230 & 5.468453 & 3.251149\end{array}$

$\begin{array}{lllll}1 & 0.222486 & 4.923160 & 4.061255\end{array}$

$\begin{array}{lllll}6 & 0.948411 & 6.832948 & 3.383291\end{array}$

$10.666505 \quad 7.358997 \quad 4.292671$

$\begin{array}{lllll}7 & 1.523327 & 7.572701 & 2.424179\end{array}$

$\begin{array}{llll}6 & 1.903321 & 6.945793 & 1.300022\end{array}$

$12.354493 \quad 7.582548 \quad 0.544102$

$\begin{array}{llll}6 & 1.691076 & 5.586194 & 1.080744\end{array}$

$12.002208 \quad 5.1323620 .146956$

$\begin{array}{llll}6 & -2.480989 & 1.809621 & 1.730578\end{array}$

$\begin{array}{llll}6 & -2.861150 & 3.161113 & 2.069628\end{array}$

$\begin{array}{llll}1 & -3.860221 & 3.489626 & 2.315075\end{array}$

$\begin{array}{lllll}7 & -1.741398 & -1.121267 & 1.499371\end{array}$

$\begin{array}{llll}6 & -3.901948 & -1.721715 & 2.079044\end{array}$

$\begin{array}{llll}1 & -4.948322 & -1.626222 & 2.325966\end{array}$

$\begin{array}{llll}6 & -3.004957 & -0.633254 & 1.754796\end{array}$

$\begin{array}{lllll}6 & -3.374223 & 0.726054 & 1.802984\end{array}$

$\begin{array}{lllll}6 & -4.818195 & 1.043100 & 2.056403\end{array}$

$\begin{array}{lllll}6 & -5.468453 & 0.696230 & 3.251149\end{array}$

$\begin{array}{lllll}1 & -4.923160 & 0.222486 & 4.061255\end{array}$

$\begin{array}{lllll}6 & -6.832948 & 0.948411 & 3.383291\end{array}$

$\begin{array}{lllll}1 & -7.358997 & 0.666505 & 4.292671\end{array}$

$\begin{array}{lllll}7 & -7.572701 & 1.523327 & 2.424179\end{array}$

$\begin{array}{llll}6 & -6.945793 & 1.903321 & 1.300022\end{array}$

$\begin{array}{llll}1 & -7.582548 & 2.354493 & 0.544102\end{array}$

$\begin{array}{lllll}6 & -5.586194 & 1.691076 & 1.080744\end{array}$

$\begin{array}{llll}1 & -5.132362 & 2.002208 & 0.146956\end{array}$

$6-0.775738-11.749100-0.047452$

$6-1.347723-13.020400 \quad 0.618478$ 
$6-0.767618-14.291390-0.019510$

$\begin{array}{lllll}6 & -1.383987 & -10.487010 & 0.547350\end{array}$

$8-2.112699-9.736051-0.079622$

$8-1.030077-10.288850 \quad 1.823800$

$1-1.059689-11.738300-1.104470$

$1-2.441462-13.017140 \quad 0.530782$

$1-1.113883-12.998210 \quad 1.690695$

$1-1.104269-14.357730-1.064258$

$\begin{array}{lllll}1 & -1.161172 & -15.178090 & 0.493291\end{array}$

$\begin{array}{lllll}6 & 0.775738 & -11.749100 & 0.047452\end{array}$

$\begin{array}{lllll}6 & 1.347723 & -13.020400 & -0.618478\end{array}$

$\begin{array}{lllll}6 & 0.767618 & -14.291390 & 0.019510\end{array}$

$\begin{array}{lllll}6 & 1.383987 & -10.487010 & -0.547350\end{array}$

$\begin{array}{lllll}8 & 2.112699 & -9.736051 & 0.079622\end{array}$

$81.030077-10.288850-1.823800$

$11.059689-11.738300 \quad 1.104470$

$12.441462-13.017140-0.530782$

$11.113883-12.998210-1.690695$

$11.104269-14.357730 \quad 1.064258$

$1 \quad 1.161172-15.178090-0.493291$

$\begin{array}{lllll}1 & -1.354317 & -9.389939 & 2.121451\end{array}$

$\begin{array}{lllll}1 & 1.354317 & -9.389939 & -2.121451\end{array}$

$6 \quad 1.809621-2.480989-1.730578$

$63.161113-2.861150-2.069628$

$13.489626-3.860221-2.315075$

$\begin{array}{lllll}7 & -1.121267 & -1.741398 & -1.499371\end{array}$

$6-1.721715-3.901948-2.079044$

$\begin{array}{llll}1 & -1.626222 & -4.948322 & -2.325966\end{array}$

$\begin{array}{llll}6 & -0.633254 & -3.004957 & -1.754796\end{array}$

$\begin{array}{lllll}6 & 0.726054 & -3.374223 & -1.802984\end{array}$

$61.043100-4.818195-2.056403$

$\begin{array}{lllll}6 & 0.696230 & -5.468453 & -3.251149\end{array}$

$10.222486-4.923160-4.061255$

$\begin{array}{lllll}6 & 0.948411 & -6.832948 & -3.383291\end{array}$

$10.666505-7.358997-4.292671$

$\begin{array}{lllll}7 & 1.523327 & -7.572701 & -2.424179\end{array}$

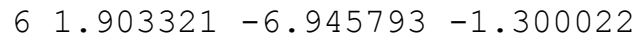

$12.354493-7.582548-0.544102$

$\begin{array}{lllll}6 & 1.691076 & -5.586194 & -1.080744\end{array}$

$12.002208-5.132362-0.146956$

$\begin{array}{lll}6 & 2.480989 & 1.809621-1.730578\end{array}$

$\begin{array}{lllll}6 & 2.861150 & 3.161113 & -2.069628\end{array}$

$13.8602213 .489626-2.315075$

$\begin{array}{lllll}7 & 1.741398 & -1.121267 & -1.499371\end{array}$

$63.901948-1.721715-2.079044$

$14.948322-1.626222-2.325966$

$\begin{array}{lllll}6 & 3.004957 & -0.633254 & -1.754796\end{array}$

$\begin{array}{lllll}6 & 3.374223 & 0.726054 & -1.802984\end{array}$

$\begin{array}{lllll}6 & 4.818195 & 1.043100 & -2.056403\end{array}$

$\begin{array}{lllll}6 & 5.468453 & 0.696230 & -3.251149\end{array}$

$\begin{array}{lllll}1 & 4.923160 & 0.222486 & -4.061255\end{array}$

$\begin{array}{llllll}6 & 6.832948 & 0.948411 & -3.383291\end{array}$

$\begin{array}{lllll}1 & 7.358997 & 0.666505 & -4.292671\end{array}$

$\begin{array}{lllll}7 & 7.572701 & 1.523327 & -2.424179\end{array}$

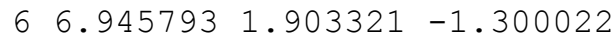

$17.5825482 .354493-0.544102$

$\begin{array}{lllll}6 & 5.586194 & 1.691076 & -1.080744\end{array}$

$15.1323622 .002208-0.146956$

$\begin{array}{lllll}6 & -1.809621 & 2.480989 & -1.730578\end{array}$

$\begin{array}{lllll}6 & -3.161113 & 2.861150 & -2.069628\end{array}$
$1-3.489626 \quad 3.860221-2.315075$

$\begin{array}{llll}7 & 1.121267 & 1.741398 & -1.499371\end{array}$

$\begin{array}{lllll}6 & 1.721715 & 3.901948 & -2.079044\end{array}$

$11.626222 \quad 4.948322-2.325966$

$\begin{array}{lllll}6 & 0.633254 & 3.004957 & -1.754796\end{array}$

$\begin{array}{lllll}6 & -0.726054 & 3.374223 & -1.802984\end{array}$

$6-1.043100 \quad 4.818195-2.056403$

$6-0.696230 \quad 5.468453-3.251149$

$1-0.222486 \quad 4.923160-4.061255$

$\begin{array}{lllll}6 & -0.948411 & 6.832948 & -3.383291\end{array}$

$1-0.666505 \quad 7.358997-4.292671$

$\begin{array}{lllll}7 & -1.523327 & 7.572701 & -2.424179\end{array}$

$6-1.903321 \quad 6.945793-1.300022$

$1-2.354493 \quad 7.582548-0.544102$

$\begin{array}{lllll}6 & -1.691076 & 5.586194 & -1.080744\end{array}$

$1-2.002208 \quad 5.132362-0.146956$

$6-2.480989-1.809621-1.730578$

$6-2.861150-3.161113-2.069628$

$1-3.860221-3.489626-2.315075$

$\begin{array}{lllll}7 & -1.741398 & 1.121267 & -1.499371\end{array}$

$\begin{array}{llll}6 & -3.901948 & 1.721715 & -2.079044\end{array}$

$1-4.9483221 .626222-2.325966$

$\begin{array}{lllll}6 & -3.004957 & 0.633254 & -1.754796\end{array}$

$6-3.374223-0.726054-1.802984$

$6-4.818195-1.043100-2.056403$

$6-5.468453-0.696230-3.251149$

$1-4.923160-0.222486-4.061255$

$6-6.832948-0.948411-3.383291$

$1-7.358997-0.666505-4.292671$

$\begin{array}{lllll}7 & -7.572701 & -1.523327 & -2.424179\end{array}$

$6-6.945793-1.903321-1.300022$

$1-7.582548-2.354493-0.544102$

$6-5.586194-1.691076-1.080744$

$1-5.132362-2.002208-0.146956$

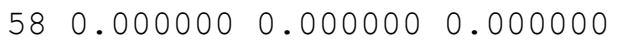

$\begin{array}{lllll}6 & 11.749100 & -0.775738 & -0.047452\end{array}$

$\begin{array}{llll}6 & 13.020400 & -1.347723 & 0.618478\end{array}$

$\begin{array}{lllll}6 & 14.291390 & -0.767618 & -0.019510\end{array}$

$\begin{array}{lllll}6 & 10.487010 & -1.383987 & 0.547350\end{array}$

$\begin{array}{llll}8 & 9.736051 & -2.112699 & -0.079622\end{array}$

$810.288850-1.030077 \quad 1.823800$

$111.738300-1.059689-1.104470$

$\begin{array}{lllll}1 & 13.017140 & -2.441462 & 0.530782\end{array}$

$112.998210-1.1138831 .690695$

$114.357730-1.104269-1.064258$

$1 \quad 15.178090-1.161172 \quad 0.493291$

$\begin{array}{lllll}6 & 11.749100 & 0.775738 & 0.047452\end{array}$

$\begin{array}{lllll}6 & 13.020400 & 1.347723 & -0.618478\end{array}$

$\begin{array}{llll}6 & 14.291390 & 0.767618 & 0.019510\end{array}$

$\begin{array}{llllll}6 & 10.487010 & 1.383987 & -0.547350\end{array}$

$\begin{array}{lllll}8 & 9.736051 & 2.112699 & 0.079622\end{array}$

$8 \quad 10.288850 \quad 1.030077-1.823800$

$\begin{array}{llll}1 & 11.738300 & 1.059689 & 1.104470\end{array}$

$1 \quad 13.017140 \quad 2.441462-0.530782$

$112.998210 \quad 1.113883-1.690695$

114.3577301 .1042691 .064258

$\begin{array}{lllll}1 & 15.178090 & 1.161172 & -0.493291\end{array}$

$\begin{array}{llll}1 & 9.389939 & 1.354317 & -2.121451\end{array}$

$\begin{array}{llll}1 & 9.389939 & -1.354317 & 2.121451\end{array}$

$\mathrm{E}(\mathrm{RB}+\mathrm{HF}-\mathrm{LYP})=-6880.76310920 \quad \mathrm{~A} . \mathrm{U}$. 


\section{References}

(S1) (a) Tashiro, K.; Konishi, K.; Aida, T. Angew. Chem. Int. Ed. Engl. 1997, 36, 856. (b) Tashiro, K.; Fujiwara, T.; Konishi, K.; Aida, T. Chem. Commun. 1998, 1121. (c) Tashiro, K.; Konishi K.; Aida, T. J. Am. Chem. Soc. 2000, 122, 7921. 